\title{
Discharge initiation by ICRF antenna in IShTAR
}

\author{
Matěj Tripskýn ${ }^{1,2, \star}$, Tom Wauters ${ }^{1}$, Rodolphe D'Inca ${ }^{3}$, Kristel Crombé ${ }^{1,2}$, Jonathan Jacquot ${ }^{3}$, Roman Ochoukov ${ }^{3}$, \\ Fabrice Louche ${ }^{1}$, Mariia Usoltceva ${ }^{2,3,4}$, Ana Kostic ${ }^{2,3}$, Anatoli Lyssoivan'1, Jean-Marie Noterdaeme ${ }^{2,3}$, and Michael \\ Van Schoor ${ }^{1}$ \\ ${ }^{1}$ Laboratory for Plasma Physics, ERM/KMS, 1000 Brussels, Belgium \\ ${ }^{2}$ Ghent University, Department of Applied Physics, 9000 Ghent, Belgium \\ ${ }^{3}$ Max-Planck-Institut für Plasmaphysik, Boltzmannstr. 2, 85748 Garching, Germany \\ ${ }^{4}$ Université de Lorraine, 54506 Vandœuvre-lés-Nancy, France
}

\begin{abstract}
IShTAR is a linear magnetized plasma test facility dedicated to the investigation of RF wave/plasma interaction. The IShTAR ICRF system consists of a single strap RF antenna. When using the antenna for plasma production without an external plasma source, it is shown that the plasma is either produced in front of the antenna strap or inside the antenna box depending on the antenna parameters. Here, we present experimental and numerical investigation of the plasma initiation parametric dependencies. Detailed pressure and RF power scans were performed in helium at $f=5.22 \mathrm{MHz}$ and $f=42.06 \mathrm{MHz}$. The experiment shows the parameter ranges for which the plasma is produced in front of the strap, or inside the antenna box. These ranges are validated by simulations with the RFdinity model, and by theoretical predictions.
\end{abstract}

\section{Introduction}

Using the ICRF antennas for discharge initiation brings the problem that the antenna load before plasma breakdown is weak. Therefore, until the plasma is created in the vessel, a high voltage is located on the antenna straps. When the plasma is formed, this voltage decreases together with an increase in the coupled power. This moment indicates the plasma breakdown. For antenna safety reasons, it is important to set the antenna parameters (power, frequency, and phasing) and the neutral pressure to minimize the breakdown time. Such optimization reduces the likelihood for the sparking inside of the antenna box.

The dependency of the breakdown time (or ionization rate) on the antenna parameters and the neutral gas pressure on Tokamaks is subject of ongoing research [1]. Simulation results have shown qualitative agreement with the experimental observations for the neutral pressure and antenna power dependency. They indicate that above a certain value of the antenna power, the breakdown times does not change with increasing power. Therefore, a first optimization consist of lowering the antenna power to avoid very high voltages. On the other hand, the neutral pressure scans demonstrate a decreasing dependency of the breakdown time with increasing pressure. For that reason, a maximum allowed pressure should be set for ICRF plasma initiation. This maximum allowed pressure is typically around $5 \cdot 10^{-2} \mathrm{~Pa}$. The simulations in [1] show that the ionization rate decreases rapidly with antenna frequency and circumference length. These dependencies may be of con-

\footnotetext{
^e-mail: matej.tripsky@rma.ac.be
}

cern for plasma production in ITER. Its ICRF system operates at high frequencies ( $f=40-55 \mathrm{MHz}$ ), and the circumference of the ITER torus is much longer than any presentday fusion machine. Nevertheless, simulations with ITER ICRF vacuum electric fields demonstrated that plasma initiation is possible even at these high frequencies and large circumference [2]. The simulations also showed that the plasma first appears close to the antenna straps. Therefore, it is crucial to understand better our set of safety conditions for the pressure, frequency and antenna power in order to avoid any possibility of plasma formation inside the ITER ICRF antenna.

The IShTAR device provides an excellent opportunity to focus on specific aspects of ICRF plasma production. It is hereto important to highlight that the IShTAR is a linear device. It can therefor be used to investigate the plasma formation in ponderomotive wells in front of the antenna box, or inside of the antenna. In toroidal devices, electrons leaving the antenna region can ionize the neutral gas along the magnetic field line and afterward they can again reenter the antenna region.

\section{Experiment on IShTAR}

The IShTAR (Ion cyclotron Sheath Test ARrangement) project focuses on studying antenna near-fields and RF sheath effects in the presence of a plasma and magnetic field [3]. It is a linear device with a cylindrical main vessel of $1.1 \mathrm{~m}$ length and $1 \mathrm{~m}$ diameter. The magnetic field is created by two coils around the main vessel. The experiment was operated with the current in the coils of 


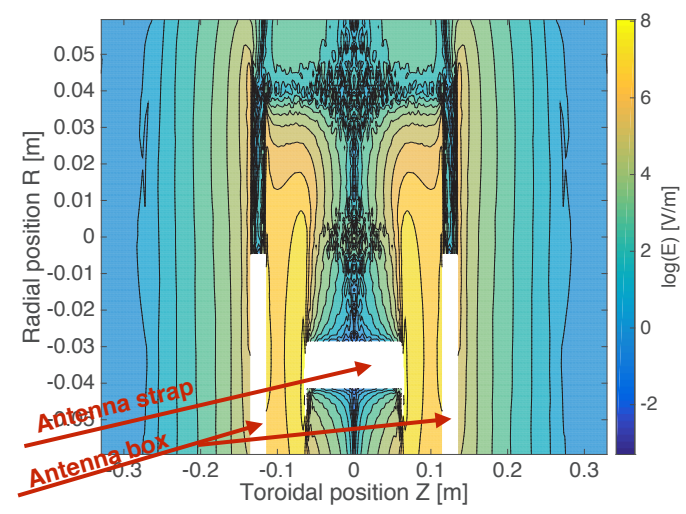

Figure 1: Contour plot of the absolute value of the parallel $E_{z}$-field in (R-Z)-coordinates for one-strap IShTAR antenna obtained in the horizontal plane using the commercial electromagnetic software CST Microwave Studio $^{\circledR}$ (MWS) for $f=5 \mathrm{MHz}, P=1 \mathrm{~W}$.

$2 \mathrm{kA}$, corresponding to the magnetic field of 0.06T. For the presented experiment plasma was initiated solely by the IShTAR ICRF antenna system. The antenna consists of a single strap fed by transmission lines connected to a generator with a power up to $1 \mathrm{~kW}$ and with a frequency range $[0.1-100 \mathrm{MHz}]$. We operated the IShTAR antenna at two frequencies, $f=5.22 \mathrm{MHz}$ and $f=42.06 \mathrm{MHz}$.

\section{Simulations}

The Monte Carlo collision model RFdinity1d3v [1] simulates the motion of electrons along a parallel magnetic field line. The model includes inelastic collisions (excitation and ionization) and elastic scattering between electrons and helium atoms. Electrons interact with the vacuum parallel electric field $E_{z}$. Depending on the electric field and frequency, electrons can be trapped in the potential well in front of the antenna strap [4]. If electrons remain trapped for a sufficient time compared to the ionisation characteristic time, the electron density may increase. The trapped electrons must be accelerated by the electric field (the generator input power) above the ionization potential $\left(\Delta E^{\text {ion }} \approx 24.6 \mathrm{eV}\right.$ for helium). Furthermore, for trapping the electrons, the electric field must have the shape (depending on the radial position) to minimize the electron losses. The performed simulations use the parallel electric field profiles as obtained by the commercial electromagnetic software CST Microwave Studio ${ }^{\circledR}$ [5]. Figure 1 shows the absolute values of the $E_{z}$-field in the horizontal plane $0.5 \mathrm{~cm}$ below the antenna top for $f=5 \mathrm{MHz}$, $P=1 \mathrm{~W}$. The figure demonstrates that not only the amplitude of the electric field changes radially but also the shape. We investigate plasma initiation at two radial locations: $R=-5.95 \mathrm{~cm}$ (inside the antenna box) and $R=5.95 \mathrm{~cm}$ (in front of the antenna strap). The vacuum parallel electric field for these two radial positions are visualized in figure 2 for a coupled power of $P=1 \mathrm{~W}$ and frequency $f=5 \mathrm{MHz}$. Two arrows indicate locations of the antenna box walls marked by the abrupt change of the

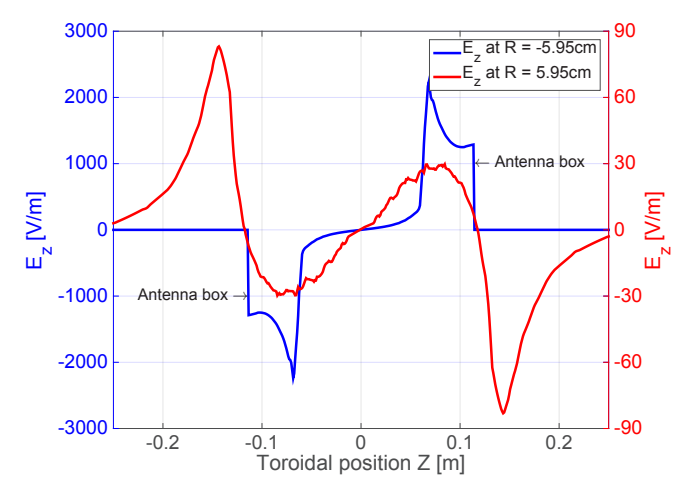

Figure 2: Vacuum $E_{z}$-field at $R=-5.95 \mathrm{~cm}$ (inside the antenna box) and $R=5.95 \mathrm{~cm}$ (in front of the antenna strap) used by the model ( $f=5 \mathrm{MHz}, P=1 \mathrm{~W})$.

$E_{z}$-field profile for the electric field inside the antenna box $(R=-5.95 \mathrm{~cm})$.

\subsection{Plasma initiation at $f=5 \mathrm{MHz}$}

Simulations were launched at $f=5 \mathrm{MHz}$ with varying neutral gas pressure and coupled power. The simulations show that the ionization rate has a linear dependency on the neutral gas pressure as predicted by Schüller [6], $v_{\text {ion }}^{\mathrm{RF}} \sim n_{\mathrm{He}}$. By fitting this linear dependence, we identify the minimum pressure above which the ionization rate is higher than zero. In the experiment, the discharges at $f=5.22 \mathrm{MHz}$ were initiated in front of the strap and never in the antenna box. The experiment demonstrated that with the increasing generator power the minimum neutral gas pressure for plasma initiation decreases. Figure 3 indicates that the minimum pressure decreases steeply at lower generator power $(100-150 \mathrm{~W})$ whereafter the minimum pressure decreases more slowly up to the maximum operated power on IShTAR $\left(P_{G}=400 \mathrm{~W}\right)$.

In the figure, the coupled power range used in the simulations is very different from the generator power range. The electric fields obtained from the MWS do not take into account eventual power losses in the matching system or transmission lines. Therefore, it is hard to link the generator power in the experiment and the coupled power from the MWS. No measurements of the voltages on the antenna strap were available. The coupled power $P=100 \mathrm{~kW}$ corresponds to the electric field amplitude $E_{0} \approx 24 \mathrm{kV} / \mathrm{m}$ at $R=5.95 \mathrm{~cm}$ for $f=5 \mathrm{MHz}$, which from experience appears to be a realistic field strength in IShTAR device. The minimum pressures in our simulations are slightly higher than the experimental ones. This shift towards the higher minimum pressures in the simulations may also be caused by the fact that our model contains only one neutral gas type, i.e. helium atom. However, the base pressure in IShTAR is $10^{-4} \mathrm{~Pa}$. It suggests that a high level of impurities can be present in the vessel $\left(\mathrm{H}_{2} \mathrm{O}\right)$ with significantly lower ionization potentials than for the helium atom. 


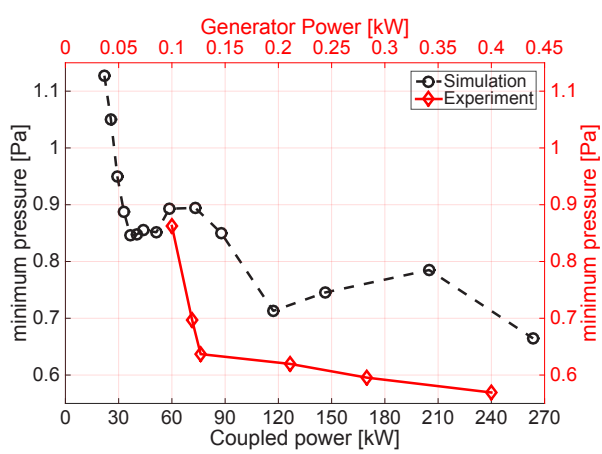

Figure 3: Dependence of the minimum pressure on the power in the experiment at $f=5.22 \mathrm{MHz}$ (red line) with the generator power and the simulations at $f=5 \mathrm{MHz}$ (black line) with the coupled power.

\subsection{Plasma initiation at $f=42 \mathrm{MHz}$}

Unlike at $f=5.22 \mathrm{MHz}$, at $f=42.06 \mathrm{MHz}$ the plasma was initiated inside the antenna box. This difference between the plasma column and its location is shown in figure 6. The figure illustrates two images from a camera on IShTAR facing the ICRF antenna for identical generator power $\left(P_{G}=290 \mathrm{~W}\right)$. At $f=5.22 \mathrm{MHz}$ the plasma column is homogeneous and located in front of the antenna, whereas at $f=42.06 \mathrm{MHz}$ the plasma is also created inside the box near the feeding point (the brightest light on the top). To understand this occurrence we reinvestigate the frequency and pressure dependency for trapping electrons in ponderomotive well to initiate the electron density build-up. The first approximation of the plasma initiation by ICRF antenna was introduced by Lyssoivan and Carter [7]. They derived conditions for the electric field and frequency to accelerate electrons above the ionization potential in the RF field. The model was later on improved by Schüller to include also the neutral pressure dependency [6]. The comparison of the theoretical predictions and simulations results for the minimum pressure dependency on the coupled power for the plasma at $f=42 \mathrm{MHz}$ are plotted in figure 4. According to the theoretical predictions and simulations results the plasma should not exist in the antenna box below $5 \mathrm{~Pa}$ at $f=42.06 \mathrm{MHz}$. Nevertheless, we observed in the experiment the plasma formation in the antenna box for the neutral gas pressures $p_{H e}>0.2 \mathrm{~Pa}$ for the medium generator power $P=290 \mathrm{~W}$.

This discrepancy between the experiment and theoretical or simulations predictions shows that we neglected in the model and theory an effect contributing in the electron multiplication in the antenna box. One of the candidates was the secondary electron emission due to electrons hitting the antenna box walls. We have implemented the secondary electron emission into our model using the yield dependency on the incoming energy from [8]. The secondary electron yield (SEY) depends strongly on the surface properties (roughness, cleanness, etc.) rather than on the material type. Therefore, we studied in more de-

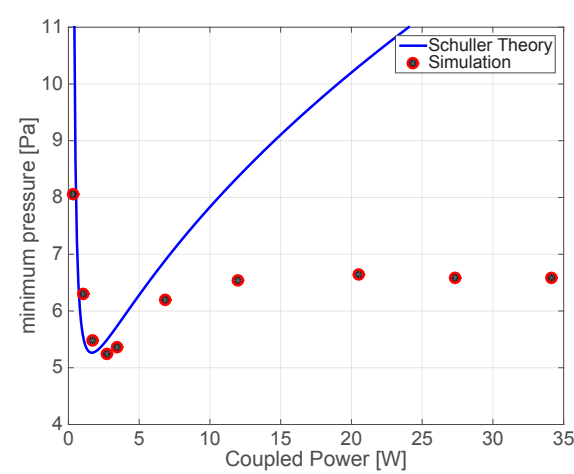

Figure 4: Comparison of the minimum pressure dependency on the power in the simulations and theoretical predictions (Schüller theory) for $f=42 \mathrm{MHz}$, and ( $W_{\text {ANT }}+$ $\left.L_{z}\right)=0.48 \mathrm{~m}$.

tails two cases: (i) low SEY with the maximum yield of 0.65 , and (ii) high SEY with the maximum yield of 2.16. The SEY energy dependencies for these two scenarios are plotted in Figure 5(C). Figures 5(A-B) show the electron density evolution for simulations inside the antenna box for these two scenarios (High and Low SEY) with the same electric field strength for the experimental frequencies $f=5 \mathrm{MHz}$, and $f=42 \mathrm{MHz}$ at different neutral pressure levels. Figure 5(A) for the case with the high SEY confirms the experimental observations that the plasma is created in the antenna box only for $f=42 \mathrm{MHz}$ even at $p_{H e}=0.2 \mathrm{~Pa}$. Whereas for $f=5 \mathrm{MHz}$, the electron density is not increasing even at the extremely high-pressure $p=8 \mathrm{~Pa}$. For the low SEY scenario, we didn't observe the electron density increase for both frequencies. The results

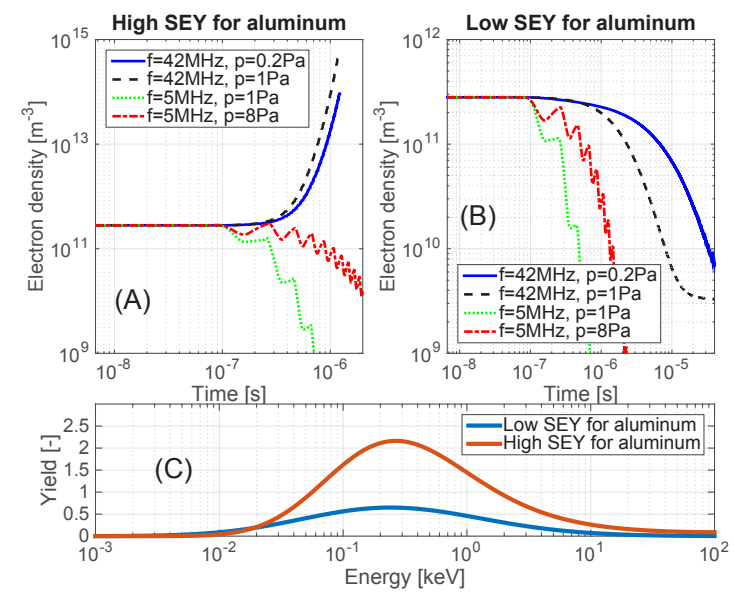

Figure 5: Electron density evolution in time for simulations with the same electric field strength at $f=5 \mathrm{MHz}$, $f=42 \mathrm{MHz}$, and various pressure levels for two scenarios with different SEY profile: (A)-High SEY, (B)-Low SEY for aluminum. (C) The SEY energy dependency for aluminum. 


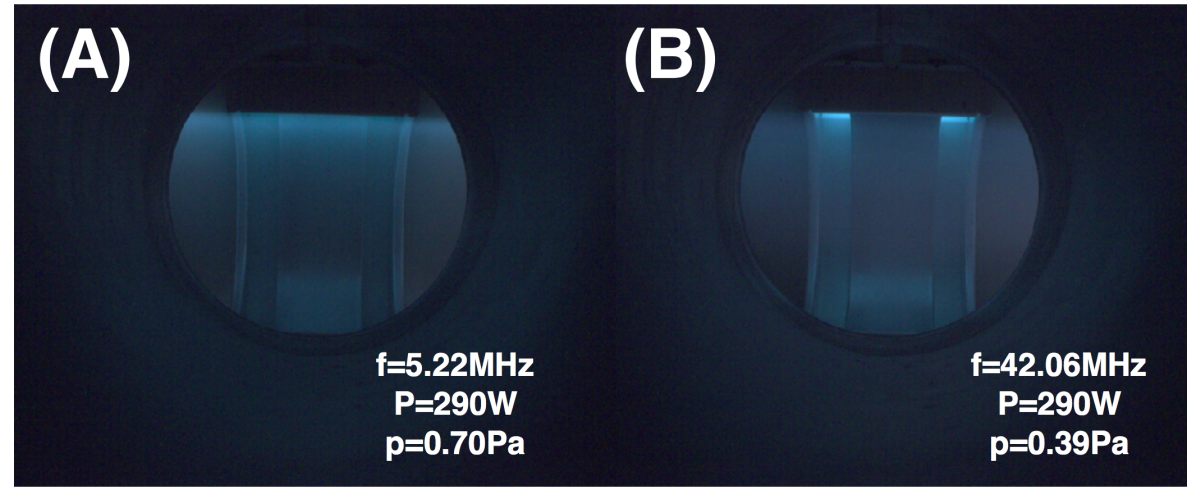

Figure 6: Camera views in IShTAR on the front side of the ICRF antenna for two frequencies at the generator power $P_{G}=290 \mathrm{~W}:$ (A) plasma is located in front of the strap for $f=5.22 \mathrm{MHz}, p_{H e}=0.7 \mathrm{~Pa},(\mathrm{~B})$ plasma is created in antenna box for $f=42.06 \mathrm{MHz}, p_{H e}=0.39 \mathrm{~Pa}$.

suggest a strong dependency not only on the frequency but the SEY energy profile as well. By implementing high but, realistic energy dependent yields from literature one easily can evoke enough multiplications to overcome particle losses at the antenna box wall.

\section{Conclusion}

A first experimental study of ICRF plasma initiation in Helium in IShTAR for two frequencies is presented. The experimental observations were compared with modeling and theoretical predictions. In the experiment, the homogeneous plasma column in front of the antenna was formed only at $f=5.22 \mathrm{MHz}$. The minimum pressures above which the plasma was created depend on the generator power and are in qualitative agreement with the modeling predictions. However, the experimental values of the minimum pressures are slightly shifted towards lower pressures. This shift may be caused by insufficient vacuum conditions or significant power losses in transmission lines and a matching unit. Impurities presented in the vessel with lower ionization potential than for the helium could increase the ionization rate. Our model (RFdinity1d3v) in the current version is not able to simulate a gas mixture.

For experiments at $f=42.06 \mathrm{MHz}$, the plasma is always created in the antenna box instead of in front of the antenna strap. The plasma was observed in the antenna box starting from pressures above $0.2 \mathrm{~Pa}$ for the generator power $P_{G}=290 \mathrm{~W}$. This observation meet the modeling predictions only after including the secondary electron emission into the model. The simulations show that the density increase in the antenna box for the given input power is possible only for $f=42 \mathrm{MHz}$ and not for $f=5 \mathrm{MHz}$ with the high SEY. It demonstrates that the secondary emission plays a crucial role in the discharge initiation inside the antenna box. The plasma inside the box was observed in the ITER ICRF frequency range. Thus more accurate model and further investigations are needed to carefully set the operation pressure limit in order to avoid plasma initiation in the antenna box in ITER.

\section{Acknowledgments}

We acknowledge the CINECA award under the ISCRA initiative, for the availability of high performance computing resources and support. This work has been carried out within the framework of the EUROfusion Consortium and has received funding from the Euratom research and training program 2014-2018 under grant agreement No 633053. The views and opinions expressed herein do not necessarily reflect those of the European Commission.

\section{References}

[1] M. Tripsky et al., European Conference Abstracts (ECA), 38F, P1.133, Berlin, Germany, (2014)

[2] M. Tripsky et al., AIP Conference Proceedings, 1689, 060009, California, USA (2015)

[3] K. Crombé et al., AIP Conference Proceedings, 1689, 030006, California, USA (2015)

[4] T. Wauters, M. Tripsky, Proc. $22^{\text {nd }} R F$ topical conference, this conference (2017)

[5] CST AG, http://www.cst.com, CST STUDIO SUITE (R) (2015)

[6] F. Schüller, Report on Applications of ICWC on ITER, Technical Report IO/2009/ADM-014 report version 3, p. 16-23, ITER - IO, 13115, St. Paul-lez-Durance, France, (2009)

[7] M.D. Carter, A.I. Lyssoivan and et al. Nuclear Fusion, 30, 723, (1990)

[8] D.C. Joy A database of electron-solid interactions, EM Facility, University of Tennessee, and Oak Ridge National Laboratory, (2008) 